\title{
Unbalanced and Minimal Point Equivalent Estimation \\ Second-Order Split-Plot Designs
}

\author{
Peter A. Parker \\ National Aeronautics and Space Administration \\ Hampton, VA 23681 \\ Scott M. Kowalski \\ Minitab Inc. \\ State College, PA 16801 \\ G. Geoffrey Vining \\ Virginia Polytechnic Institute and State University \\ Blacksburg, VA 24061-0439
}

\begin{abstract}
Restricting the randomization of hard-to-change factors in industrial experiments is often performed by employing a split-plot design structure. From an economic perspective, these designs minimize the experimental cost by reducing the number of resets of the hard-tochange factors. In this paper, unbalanced designs are considered for cases where the subplots are relatively expensive and the experimental apparatus accommodates an unequal number of runs per whole-plot. We provide construction methods for unbalanced second-order splitplot designs that possess the equivalence estimation optimality property, providing best linear unbiased estimates of the parameters; independent of the variance components. Unbalanced versions of the central composite and Box-Behnken designs are developed. For cases where the subplot cost approaches the whole-plot cost, minimal point designs are proposed and illustrated with a split-plot Notz design.
\end{abstract}

KEY WORDS: Box-Behnken Design, Central Composite Design, Equivalent Estimation Design, Notz Design, Restricted Randomization, Split-Plot Design.

Dr. Parker is an Aerospace Electronics Engineer in the Aeronautics Systems Engineering Branch at Langley Research Center. He is a Member of ASQ. His email address is peter.a.parker@larc.nasa.gov

Dr. Kowalski is a Technical Trainer. He is a Senior Member of ASQ. His email address is skowalski@minitab.com.

Dr. Vining is Professor and Head of the Department of Statistics. He is a Fellow of ASQ. His email address is vining@vt.edu. 


\section{Introduction}

Frequently, industrial experiments involve factors that are difficult, time consuming, or costly to manipulate, referred to as hard-to-change (HTC) factors. Alternatively, some factors may be relatively easy to manipulate, referred to as easy-to-change (ETC) factors. A split-plot design structure is an efficient experimental approach that reduces the number of settings of the HTC factors. We assign the HTC factors to the whole-plots and the ETC factors to the subplots. For a more detailed development of split-plot designs see Montgomery (2004) and Myers and Montgomery (2002).

Vining, Kowalski, and Montgomery (2005) and Parker, Kowalski, and Vining (2005a) proposed design construction techniques for balanced equivalent estimation designs. To achieve balance (same number of subplot runs in each whole-plot) in these designs, subplot runs were replicated within each whole-plot. Unbalanced versions, with different whole-plot sizes, reduce the replication of the subplot runs allowing a more efficient allocation of experimental resources at the subplot level. In addition, when the whole-plot size is large, replicating subplot runs to achieve balance generates an excessive number of subplot error variance degrees of freedom. Unbalanced designs provide attractive alternatives to practitioners when they are permissible.

In experimental design, we strive for small designs that meet our objectives and simultaneously minimize cost. Similarly in the split-plot context, the cost of the experiment is an important criteria, however the definition of small is not as clear because we have two types of experimental units with different associated costs. In many cases, the cost of the whole-plot experimental units is much greater than that of the subplot units, making the subplot runs essentially free. A balanced design is appropriate in this situation. However, if the subplot runs are relatively expensive as compared to subplot runs, then an unbalanced 
design that simultaneously minimizes the number of whole-plots and subplot experimental units is attractive from an economic perspective. Note that if the cost of the whole-plots equals the cost of the subplots, then a completely randomized design is appropriate.

In addition to the cost considerations, the experimental apparatus plays a role in determining whether an unbalanced design is a viable option. For example, if the subplot units are specimens and the whole-plots represent the settings of a temperature chamber, then varying the number of subplot experimental units may introduce an additional source of variability due to changing the thermal load in the chamber.

In aerodynamic wind tunnel research, the configuration of the scaled test article usually requires a partial mechanical disassembly, making it a HTC factor. Alternatively, the flow field parameters are relatively ETC change (see Parker, Kowalski, and Vining (2005a, 2005b)). To simulate flight conditions, there is a class of wind tunnel laboratories that operate in a cryogenic pressurized environment. In these complex facilities, the setting of a wholeplot factor condition is time consuming and expensive. This is due to the conditioning of the environment that allows personnel access the test article and perform the configuration change. Once the whole-plot conditions are set, maintaining these extreme environmental conditions over time is costly and is directly proportional to the number of subplot runs. In this example, the whole-plot size is not determined by the capacity of the experimental apparatus, rather it represents the time to execute the subplot runs. An economical design for this application simultaneously minimizes the number of whole-plots and the total number of subplot runs.

In this paper, we review the conditions under which ordinary least squares is an appropriate method of estimation for a second-order split-plot design. Based on these conditions, we illustrate two systematic design construction strategies to build unbalanced equivalent estimation designs from the central composite (CCD) and Box-Behnken (BBD). Replication of 
subplot runs within each whole-plot has been reduced over the balanced versions, thereby reducing the size of some whole-plots. This development does not attempt to generalize all of the admissible forms of unbalance. Instead, we focus on a strategy to reduce the size of the whole-plots containing subplot replicates, especially those containing replicated subplot centers. In addition, we discuss minimizing the total number of subplot runs by configuring response surface minimal point designs into an equivalent estimation split-plot structure.

The notation $\mathrm{D}(w, k)$ is used to denote a design with $w$ whole-plot (HTC) factors and $k$ subplot (ETC) factors. We use $\alpha$ and $\beta$ to denote the distance to the axial points for the subplot and whole-plot factors, respectively. We assume that the value of $\alpha$ is the same for all of the subplot factors and similarly $\beta$ is the same for all of the whole-plot factors. Designs with combinations of $1 \leq w \leq 3$ and $2 \leq k \leq 4$ are considered to encompass many practical situations. Note that unbalanced designs with $k=1$ result in whole-plots of size one and have not been included.

\section{Second-Order Split-Plot Designs}

Letsinger, Myers, and Lentner (1996) emphasized the need for research in the area of secondorder split-plot designs and focused on analysis issues; recommending the use of restricted maximum likelihood for model estimation and inference. They proposed sorting a completely randomized design (CRD) into a split-plot structure, which often results in an unbalanced design. Draper and John (1998) considered modification of the central composite design (CCD) (Box and Wilson (1951)) to be executed in a split-plot mode to achieve near-rotatability. Trinca and Gilmour (2001) studied multiple layers of split-plotting, called multistratum designs, where the two-level split-plot design is a special case of this general class. They employed a computer-intensive search algorithm to construct designs that maintained nearorthogonality between strata. Goos and Vandebroek $(2001,2003)$ proposed the use of a 
point-exchange algorithm to construct $\mathcal{D}$-optimal split-plot designs, which minimize the volume of the joint confidence region of regression coefficients. Goos (2002) provides a thorough development of balanced and unbalanced $\mathcal{D}$-optimal split-plot designs.

Vining, Kowalski, and Montgomery (2005), hereafter referred to as VKM, proposed a class of second-order SPD's where ordinary least squares (OLS) is equivalent to generalized least squares (GLS) for model estimation. Moreover, they proposed augmentation of these designs to provide pure-error estimates at both the whole-plot and subplot levels. Parker, Kowalski, and Vining (2005a) provided a generalized derivation of the equivalence conditions that leads to the development of systematic construction strategies and the ability to numerically verify if an arbitrary design achieves the equivalence property. They illustrated the construction of split-plot versions of the central composite design and a class of equivalent estimation $\mathcal{D}$-optimal designs. Parker, Kowalski, and Vining (2005b) extends the classes of designs to include balanced split-plot versions of the Box-Behnken, equiradial, and small composite design.

Equivalent estimation designs offer a number of attractive features. For example, the equivalence property is independent of the variance components. Moreover, the parameter estimates are BLUE (best linear unbiased estimators), independent of the variance components, and robust to the assumption of normality. Model estimation of equivalent estimation designs is simplified using OLS; eliminating the need for specialized software that is often times unavailable to the industrial practitioner.

\section{Equivalence Conditions}

We define the general form of the model as

$$
\mathbf{y}=\mathbf{X} \boldsymbol{\beta}+\boldsymbol{\delta}+\boldsymbol{\epsilon}
$$


where $\mathbf{y}$ is the $N \times 1$ vector of responses, $\mathbf{X}$ is the $N \times p$ model matrix with rank of $p, \boldsymbol{\beta}$ is the $p \times 1$ vector of coefficients, $\boldsymbol{\delta}$ is the $N \times 1$ vector of random whole-plot errors, $\boldsymbol{\epsilon}$ is the $N \times 1$ vector of random subplot errors, $N$ is the total number of subplot runs, and $p$ is the number of terms in the model including the intercept. We assume that $\boldsymbol{\delta}+\boldsymbol{\epsilon}$ has a mean of $\mathbf{0}$ and variance-covariance matrix $\boldsymbol{\Sigma}=\sigma_{\epsilon}^{2} \mathbf{I}+\sigma_{\delta}^{2} \mathbf{J}_{b}$, where $\sigma_{\epsilon}^{2}$ is the subplot error variance, and $\sigma_{\delta}^{2}$ is the whole-plot error variance. For an unbalanced design, $\mathbf{J}_{b}$ is a function of the $N \times p$ incidence matrix $\mathbf{Z}$ expressed as $\mathbf{J}_{b}=\mathbf{Z} \mathbf{Z}^{\prime}$.

The matrix $\mathbf{J}_{b}$ has the form of

$$
\mathbf{J}_{b}=\left[\begin{array}{cccc}
\mathbf{1}_{n_{1}} \mathbf{1}_{n_{1}}^{\prime} & 0 & \cdots & 0 \\
0 & \mathbf{1}_{n_{2}} \mathbf{1}_{n_{2}}^{\prime} & \cdots & 0 \\
\vdots & \vdots & \ddots & \vdots \\
0 & 0 & \cdots & \mathbf{1}_{n_{m}} \mathbf{1}_{n_{m}}^{\prime}
\end{array}\right]
$$

where $m$ is the number of whole-plots and $n_{i}$ is the number of subplot runs in the $i^{t h}$ wholeplot and denotes the length of the vector of ones.

Parker, Kowalski, and Vining (2005a) provide a detailed derivation of the equivalence condition, summarized as follows. We assume that there is one level of split-plotting and the design supports the specified model. The necessary and sufficient condition for equivalence from McElroy (1967) (see also Graybill (1976, p. 209)) is

$$
\mathrm{XF}=\Sigma \mathrm{X}
$$

where $\mathbf{F}$ is a $p \times p$ non-singular matrix. We define $\mathbf{F}$ to be

$$
\mathbf{F}=\sigma_{\epsilon}^{2} \mathbf{I}+\sigma_{\delta}^{2} \mathbf{K},
$$

where,

$$
\mathbf{K}=\left(\mathbf{X}^{\prime} \mathbf{X}\right)^{-1} \mathbf{X}^{\prime} \mathbf{J}_{b} \mathbf{X}
$$


It can be shown that the condition for equivalence can be expressed as

$$
\mathbf{X K}=\mathbf{J}_{b} \mathbf{X}
$$

The right-hand side of this equation sums the columns of the model matrix within each whole-plot. These column totals, or unscaled whole-plot means, must equal the model matrix multiplied by $\mathbf{K}$ to satisfy the condition of equivalence. Note that the equivalence condition is independent of the variance components, $\sigma_{\epsilon}^{2}$ and $\sigma_{\delta}^{2}$.

For a complete second-order model, let $\mathbf{X}$ be partitioned as

$$
\mathbf{X}=\left[\begin{array}{ccccc}
\mathbf{1} & \mathbf{W}_{\mathrm{D}_{1}} & \mathbf{W}_{\mathrm{Q}_{1}} & \mathbf{S}_{\mathrm{D}_{1}} & \mathbf{S}_{\mathrm{Q}_{1}} \\
\mathbf{1} & \mathbf{W}_{\mathrm{D}_{2}} & \mathbf{W}_{\mathrm{Q}_{2}} & \mathbf{S}_{\mathrm{D}_{2}} & \mathbf{S}_{\mathrm{Q}_{2}} \\
\vdots & \vdots & \vdots & \vdots & \vdots \\
\mathbf{1} & \mathbf{W}_{\mathrm{D}_{m}} & \mathbf{W}_{\mathrm{Q}_{m}} & \mathbf{S}_{\mathrm{D}_{m}} & \mathbf{S}_{\mathrm{Q}_{m}}
\end{array}\right]
$$

where the first column corresponds to the intercept, $\mathbf{W}_{\mathrm{D}_{i}}$ denotes the whole-plot main effects and two-factor interactions, $\mathbf{W}_{\mathrm{Q}_{i}}$ contains the whole-plot pure quadratics, $\mathbf{S}_{\mathrm{D}_{i}}$ includes the subplot main effects, subplot by subplot two-factor interactions, and the whole-plot by subplot interactions, and $\mathbf{S}_{\mathrm{Q}_{i}}$ denotes the subplot pure quadratic terms of the $i^{\text {th }}$ whole-plot.

Consider a form of $\mathbf{K}$ for the unbalanced design as,

$$
\mathbf{K}_{(p \times p)}=\left[\begin{array}{ccccc}
n_{0} & \mathbf{0}^{\prime} & \mathbf{0}^{\prime} & \mathbf{0}^{\prime} & \mathbf{m}_{\mathbf{0}}^{\prime} \\
\mathbf{0} & n_{\mathrm{W}} \mathbf{I}_{\mathrm{D}} & \mathbf{0} & \mathbf{0} & \mathbf{0} \\
\mathbf{u}_{\mathrm{W}} & \mathbf{0} & n_{\mathrm{W}} \mathbf{I}_{\mathrm{Q}} & \mathbf{0} & \mathbf{M}_{\mathrm{W}} \\
\mathbf{0} & \mathbf{0} & \mathbf{0} & \mathbf{0} & \mathbf{0} \\
\mathbf{u}_{\mathrm{S}} & \mathbf{0} & \mathbf{0} & \mathbf{0} & \mathbf{V}_{\mathrm{S}}
\end{array}\right] .
$$

where $n_{0}$ and $n_{\mathrm{W}}$ are scalars, $\mathbf{m}_{\mathbf{0}}$ is a vector of length $k, \mathbf{I}_{\mathrm{D}}$ and $\mathbf{I}_{\mathrm{Q}}$ are $\left(w+\frac{w(w-1)}{2}\right) \times(w+$ $\left.\frac{w(w-1)}{2}\right)$ and $w \times w$ identity matrices, respectively, $\mathbf{u}_{\mathrm{W}}$ is a vector of length $w, \mathbf{u}_{\mathrm{S}}$ is a vector of length $k, \mathbf{M}_{\mathrm{W}}$ is a $w \times k$ matrix, and $\mathbf{V}_{\mathrm{S}}$ is a $k \times k$ matrix. The definition of $\mathbf{m}_{\mathbf{0}}, \mathbf{M}_{\mathrm{W}}$, and $\mathbf{V}_{\mathrm{S}}$ were defined by Parker, Kowalski, and Vining (2005a, 2005b) for balanced designs. The proposed elements, $\mathbf{u}_{\mathrm{W}}$, and $\mathbf{u}_{\mathrm{S}}$, are specific to unbalanced designs and are defined in this paper. 
Performing the multiplication in Equation (2) we obtain

$$
\begin{aligned}
& \mathbf{X K}=\left[\begin{array}{ccccc}
\mathbf{1} & \mathbf{W}_{\mathrm{D}_{1}} & \mathbf{W}_{\mathrm{Q}_{1}} & \mathbf{S}_{\mathrm{D}_{1}} & \mathbf{S}_{\mathrm{Q}_{1}} \\
\mathbf{1} & \mathbf{W}_{\mathrm{D}_{2}} & \mathbf{W}_{\mathrm{Q}_{2}} & \mathbf{S}_{\mathrm{D}_{2}} & \mathbf{S}_{\mathrm{Q}_{2}} \\
\vdots & \vdots & \vdots & \vdots & \vdots \\
\mathbf{1} & \mathbf{W}_{\mathrm{D}_{m}} & \mathbf{W}_{\mathrm{Q}_{m}} & \mathbf{S}_{\mathrm{D}_{m}} & \mathbf{S}_{\mathrm{Q}_{m}}
\end{array}\right]\left[\begin{array}{ccccc}
n_{0} & \mathbf{0}^{\prime} & \mathbf{0}^{\prime} & \mathbf{0}^{\prime} & \mathbf{m}_{\mathbf{0}}^{\prime} \\
\mathbf{0} & n_{\mathrm{W}_{\mathrm{D}}} & \mathbf{0} & \mathbf{0} & \mathbf{0} \\
\mathbf{u}_{\mathrm{W}} & \mathbf{0} & n_{\mathrm{W}} \mathbf{I}_{\mathrm{Q}} & \mathbf{0} & \mathbf{M}_{\mathrm{W}} \\
\mathbf{0} & \mathbf{0} & \mathbf{0} & \mathbf{0} & \mathbf{0} \\
\mathbf{u}_{\mathrm{S}} & \mathbf{0} & \mathbf{0} & \mathbf{0} & \mathbf{V}_{\mathrm{S}}
\end{array}\right] \\
& \mathbf{J}_{b} \mathbf{X}=\left[\begin{array}{ccccc}
n_{1} \mathbf{1} & 11^{\prime} \mathbf{W}_{\mathrm{D}_{1}} & 11^{\prime} \mathbf{W}_{\mathrm{Q}_{1}} & 1^{\prime} \mathbf{S}_{\mathrm{D}_{1}} & 11^{\prime} \mathbf{S}_{\mathrm{Q}_{1}} \\
n_{2} \mathbf{1} & 11^{\prime} \mathbf{W}_{\mathrm{D}_{2}} & 11^{\prime} \mathbf{W}_{\mathrm{Q}_{2}} & 11^{\prime} \mathbf{S}_{\mathrm{D}_{2}} & 11^{\prime} \mathbf{S}_{\mathrm{Q}_{2}} \\
\vdots & \vdots & \vdots & \vdots & \vdots \\
n_{m} \mathbf{1} & 11^{\prime} \mathbf{W}_{\mathrm{D}_{m}} & 11^{\prime} \mathbf{W}_{\mathrm{Q}_{m}} & 11^{\prime} \mathrm{S}_{\mathrm{D}_{m}} & 11^{\prime} \mathbf{S}_{\mathrm{Q}_{m}}
\end{array}\right]
\end{aligned}
$$

where the vectors of ones have length $n_{i}$ in the $i^{\text {th }}$ whole-plot (explicit subscripts on these vectors are omitted for clarity). From Equation (4), we see that the conditions to achieve equivalence are isolated to the relationships between the within whole-plot column sums of the intercept and the whole-plot and subplot pure quadratic terms. In addition, we see by inspection that the designs possess first-order plus interaction orthogonal subplot designs.

If the design possess the equivalence property, then $\hat{\boldsymbol{\beta}}_{O L S}=\hat{\boldsymbol{\beta}}_{G L S}$ and ordinary least squares (OLS) is an appropriate method of estimation. Note that it is a rather remarkable result that OLS would be an appropriate method of estimation for a class of unbalanced second-order split-plot designs. The OLS estimate of the model coefficients is $\hat{\boldsymbol{\beta}}_{O L S}=\left(\mathbf{X}^{\prime} \mathbf{X}\right)^{-1} \mathbf{X}^{\prime} \mathbf{y}$. This expression highlights that the parameter estimates from an equivalent estimation split-plot design are independent of the variance components. By equivalence to GLS, the variancecovariance matrix for the OLS estimate is $\left(\mathbf{X}^{\prime} \Sigma^{-1} \mathbf{X}\right)^{-1}$.

\section{Balanced Design Summary}

Parker, Kowalski, and Vining (2005a) proposed two systematic design construction techniques that enable the construction of equivalent estimation SPD's derived from completely randomized response surface designs. The first technique is a generalized version of the 
Table 1: Elements of $\mathbf{K}$ for Balanced Designs.

(VKM and MWP denotes method of construction, $\mathbf{V}_{\mathrm{S}}, \mathbf{m}_{\mathbf{0}}$, and $\phi$ denote the elements of $\mathbf{K}$ )

\begin{tabular}{|c|c|c|c|}
\hline & VKM-CCD & VKM-BBD & MWP-BBD \\
\hline $\mathbf{V}_{\mathrm{S}}$ & $\frac{n}{k} \mathbf{1}_{k} \mathbf{1}_{k}^{\prime}$ & $2(k-1) \mathbf{1}_{k} \mathbf{1}_{k}^{\prime}$ & $\mathbf{0}$ \\
\hline $\mathbf{m}_{\mathbf{0}}$ & $\mathbf{0}$ & $\mathbf{0}$ & $4(k-1) \mathbf{1}_{k}$ \\
\hline$\phi$ & 0 & 0 & $6-4 k$ \\
\hline
\end{tabular}

construction method proposed by Vining, Kowalski, and Montgomery (2005), subsequently referred to as the VKM method. In the second method, we obtain designs that achieve the minimum number of whole-plots to configure a classical CCD into a split-plot structure, subsequently referred to as the MWP method. Based on the construction method and the parent completely randomized design (CRD), we define the elements of $\mathbf{K}$.

The elements of $\mathbf{K}$ in Equation (3), derived in Parker, Kowalski, and Vining (2005a, 2005b) are summarized in Table 1 . For the balanced case, $n_{0}=n_{\mathrm{W}}=n$ and is the number of subplot runs per whole-plot. We define $\mathbf{M}_{\mathrm{W}}$ to be of the form $\phi \mathbf{1}_{w} \mathbf{1}_{k}^{\prime}$. Note that the values for the VKM construction method apply to cases where the number of subplot factors is a power of 2 , and the values for the MWP method apply to $w=1$.

\section{Unbalanced VKM Method CCD}

To construct an unbalanced design, we first construct the balanced version. Then, we reduce the size of the whole-plot containing all factors at their center level. We denote the size of this whole-plot as $n_{c}$ in the derivation, and choose $n_{c}=2$ in the examples presented.

The construction of a VKM method CCD is described in Parker, Kowalski, and Vining (2005a). We begin with a completely randomized CCD in $w+k=f$ factors. The design is rearranged such that the HTC factors remain constant within each WP and the subplot 
designs are orthogonal for a first-order plus interaction model. The axial points (or star points) of the HTC factors are placed in a WP by themselves. The center points, consisting of all factors at their zero level in coded units, are also placed in a separate whole-plot. To maintain balance, subplot runs are replicated as required within each whole-plot.

For an unbalanced VKM method CCD, when $k$ is a power of 2 , we define $\mathbf{K}$ by setting $\mathbf{m}_{\mathbf{0}}=\mathbf{0}$ and $\mathbf{M}_{\mathrm{W}}=\mathbf{0}$. Based on this form of $\mathbf{K}$ and Equation 4, the conditions that must be satisfied are

$$
\begin{aligned}
n_{\mathrm{W}} \mathbf{W}_{\mathrm{D}_{i}} & =\mathbf{1}_{n_{i}} \mathbf{1}_{n_{i}}^{\prime} \mathbf{W}_{\mathrm{D}_{i}} \\
n_{\mathrm{W}} \mathbf{W}_{\mathrm{Q}_{i}} & =\mathbf{1}_{n_{i}} \mathbf{1}_{n_{i}}^{\prime} \mathbf{W}_{\mathrm{Q}_{i}} \quad \forall i
\end{aligned}
$$

and

$$
\begin{aligned}
\mathbf{1}_{n_{i}} \mathbf{1}_{n_{i}}^{\prime} \mathbf{S}_{\mathrm{Q}_{i}} & =\mathbf{S}_{\mathrm{Q}_{i}} \mathbf{V}_{\mathrm{S}} \\
n_{i} \mathbf{1}_{n_{i}} & =n_{0} \mathbf{1}_{n_{i}}+\mathbf{W}_{\mathrm{Q}_{i}} \mathbf{u}_{\mathrm{W}}+\mathbf{S}_{\mathrm{Q}_{i}} \mathbf{u}_{\mathrm{S}} \quad \forall i
\end{aligned}
$$

For the conditions in Equation (5), since whole-plot factors are held constant within each whole-plot, the column sums of the whole-plot model matrix equals $n_{i}$ times the level of the whole-plot model term. Therefore, we find $n_{\mathrm{W}}$ equals the number of subplot runs in the whole-plots containing the HTC factors at their non-zero levels. Note that for this construction method all whole-plots of this type must possess the same number of subplot runs. These whole-plot model conditions are satisfied for any design by the nature of the split-plot structure.

In Equation (6), the first condition involves the column sums of the subplot pure quadratic terms and $\mathbf{V}_{\mathrm{S}}$ is defined in Table 1. The second condition expresses a relationship between each row of the whole-plot model matrix and the number of subplot runs in that whole-plot, $n_{i}$. We denote the $j^{\text {th }}$ row of the model matrix for the whole-plot and subplot pure quadratic 
terms within the $i^{\text {th }}$ whole-plot as $\mathbf{W}_{\mathrm{Q}_{i j}}$ and $\mathbf{S}_{\mathrm{Q}_{i j}}$, respectively. We define the general form of $\mathbf{u}_{\mathrm{W}}$ to be $\lambda \mathbf{1}_{w}$ and $\mathbf{u}_{\mathrm{S}}$ to be $\rho \mathbf{1}_{k}$, where $\lambda$ and $\rho$ are suitable constants chosen to satisfy the equivalence conditions. Therefore, we express the unbalanced condition as

$$
n_{i}=n_{0}+\lambda \mathbf{W}_{\mathrm{Q}_{i j}} \mathbf{1}_{w}+\rho \mathbf{S}_{\mathrm{Q}_{i j}} \mathbf{1}_{k} \quad \forall i, j .
$$

To find expressions for $n_{0}, \lambda$, and $\rho$, we consider the forms of $\mathbf{W}_{\mathrm{Q}_{i j}}$ and $\mathbf{S}_{\mathrm{Q}_{i j}}$ for each type of whole-plot found in a CCD constructed according to the VKM method, summarized in Table 2. Note that for the whole-plot and subplot axial runs the position of $\alpha^{2}$ and $\beta^{2}$ will change depending on the factor involved, however there is always a single element in each row vector.

Table 2: VKM Method CCD Cases of Whole-Plot Type.

$\left(\mathbf{W}_{\mathrm{Q}_{i j}}\right.$ is the $j^{\text {th }}$ row of the model matrix containing the WP pure quadratics,

$\mathbf{S}_{\mathrm{Q}_{i j}}$ is the $j^{\text {th }}$ row of the model matrix containing the SP pure quadratics, $n_{i}$ is the number of subplot runs in the $i^{\text {th }}$ whole-plot, $\alpha$ and $\beta$ are the axial point distances for the ETC and HTC factors, respectively)

\begin{tabular}{|c|c|c|c|c|}
\hline Case & WP Type & $\mathbf{W}_{\mathrm{Q}_{i j}}$ & $\mathbf{S}_{\mathrm{Q}_{i j}}$ & $n_{i}$ \\
\hline $\mathrm{c}$ & all centers & {$\left[\begin{array}{lll}0 & \cdots & 0\end{array}\right]_{w}$} & {$\left[\begin{array}{lll}0 & \cdots & 0\end{array}\right]_{k}$} & $n_{c}$ \\
\hline 1 & WP centers, SP axials & {$\left[\begin{array}{lll}0 & \cdots & 0\end{array}\right]_{w}$} & {$\left[\begin{array}{cccc}\alpha^{2} & 0 & \cdots & 0\end{array}\right]_{k}$} & $n_{1}=2 k$ \\
\hline 2 & WP axials, SP centers & {$\left[\begin{array}{llll}\beta^{2} & 0 & \cdots & 0\end{array}\right]_{w}$} & {$\left[\begin{array}{lll}0 & \cdots & 0\end{array}\right]_{k}$} & $n_{2}=n_{f}$ \\
\hline 3 & factorial & {$\left[\begin{array}{lll}1 & \cdots & 1\end{array}\right]_{w}$} & {$\left[\begin{array}{lll}1 & \cdots & 1\end{array}\right]_{k}$} & $n_{3}=n_{f}$ \\
\hline
\end{tabular}

From case (c) and Equation (7), we see that $n_{0}=n_{c}$. Based on the structure of the row vectors, we can express Equation (7) for each case as

Case (1): $\quad n_{1}-n_{c}=\rho \alpha^{2}$

Case (2): $\quad n_{2}-n_{c}=\lambda \beta^{2}$ 
Case (3): $\quad n_{3}-n_{c}=\lambda w+\rho k$

In a CCD, $n_{1}=2 k$ and $n_{2}=n_{3}=n_{f}$, where $n_{f}$ denotes the number of subplot runs in the factorial whole-plots. Therefore, we find

$$
\begin{gathered}
\rho=\frac{2 k-n_{c}}{\alpha^{2}} \\
\lambda=\frac{n_{f}-n_{c}}{\beta^{2}} .
\end{gathered}
$$

In addition from case (2) and (3), we see that $\lambda \beta^{2}=\lambda w+\rho k$. Solving this expression for $\alpha$ and $\beta$ provides,

$$
\begin{array}{r}
\alpha=\sqrt{\frac{k\left(2 k-n_{c}\right)}{\left(n_{f}-n_{c}\right)\left(1-\frac{w}{\beta^{2}}\right)}} \\
\beta=\sqrt{\frac{w \alpha^{2}\left(n_{f}-n_{c}\right)}{\alpha^{2}\left(n_{f}-n_{c}\right)-k\left(2 k-n_{c}\right)}} .
\end{array}
$$

If we desire a design with $\alpha=\beta$, then we obtain

$$
\alpha=\beta=\sqrt{w+\frac{k\left(2 k-n_{c}\right)}{\left(n_{f}-n_{c}\right)}} .
$$

Performing an analysis of Equations (8) and (9), we find that our choice of $\alpha$ and $\beta$ are constrained by

$$
\begin{array}{r}
\alpha>\sqrt{\frac{k\left(2 k-n_{c}\right)}{n_{f}-n_{c}}} \\
\beta>\sqrt{w} .
\end{array}
$$

In addition from Equations (8) and (9), we see that the relationship between $\alpha$ and $\beta$ is inversely proportional. Using these expressions, we can define $\mathbf{K}$ for an unbalanced VKM CCD when $k$ is a power of 2 . 
Table 3: VKM Method Unbalanced Central Composite Design for $\mathrm{D}(2,2)$.

$\left(z_{1}\right.$ and $z_{2}$ are HTC factors, $x_{1}$ and $x_{2}$ are ETC factors, $\alpha$ and $\beta$ are the distances to the axial points for the ETC and HTC factors, respectively)

\begin{tabular}{|c|c|c|c|c|c|c|c|c|c|}
\hline Whole-Plot & $z_{1}$ & $z_{2}$ & $x_{1}$ & $x_{2}$ & Whole-Plot & $z_{1}$ & $z_{2}$ & $x_{1}$ & $x_{2}$ \\
\hline \multirow[t]{4}{*}{1} & -1 & -1 & -1 & -1 & \multirow[t]{4}{*}{6} & $\beta$ & 0 & 0 & 0 \\
\hline & -1 & -1 & 1 & -1 & & $\beta$ & 0 & 0 & 0 \\
\hline & -1 & -1 & -1 & 1 & & $\beta$ & 0 & 0 & 0 \\
\hline & -1 & -1 & 1 & 1 & & $\beta$ & 0 & 0 & 0 \\
\hline \multirow[t]{4}{*}{2} & 1 & -1 & -1 & -1 & \multirow[t]{4}{*}{7} & 0 & $-\beta$ & 0 & 0 \\
\hline & 1 & -1 & 1 & -1 & & 0 & $-\beta$ & 0 & 0 \\
\hline & 1 & -1 & -1 & 1 & & 0 & $-\beta$ & 0 & 0 \\
\hline & 1 & -1 & 1 & 1 & & 0 & $-\beta$ & 0 & 0 \\
\hline \multirow[t]{4}{*}{3} & -1 & 1 & -1 & -1 & \multirow[t]{4}{*}{8} & 0 & $\beta$ & 0 & 0 \\
\hline & -1 & 1 & 1 & -1 & & 0 & $\beta$ & 0 & 0 \\
\hline & -1 & 1 & -1 & 1 & & 0 & $\beta$ & 0 & 0 \\
\hline & -1 & 1 & 1 & 1 & & 0 & $\beta$ & 0 & 0 \\
\hline \multirow[t]{4}{*}{4} & 1 & 1 & -1 & -1 & \multirow[t]{4}{*}{9} & 0 & 0 & $-\alpha$ & 0 \\
\hline & 1 & 1 & 1 & -1 & & 0 & 0 & $\alpha$ & 0 \\
\hline & 1 & 1 & -1 & 1 & & 0 & 0 & 0 & $-\alpha$ \\
\hline & 1 & 1 & 1 & 1 & & 0 & 0 & 0 & $\alpha$ \\
\hline \multirow[t]{4}{*}{5} & $-\beta$ & 0 & 0 & 0 & \multirow[t]{4}{*}{10} & 0 & 0 & 0 & 0 \\
\hline & $-\beta$ & 0 & 0 & 0 & & 0 & 0 & 0 & 0 \\
\hline & $-\beta$ & 0 & 0 & 0 & & & & & \\
\hline & $-\beta$ & 0 & 0 & 0 & & & & & \\
\hline
\end{tabular}

Consider a split-plot design with two hard-to-change factors, denoted by $z_{1}$ and $z_{2}$, and two easy-to-change factors, denoted by $x_{1}$ and $x_{2}$. An unbalanced central composite design (CCD) in coded units is provided in Table 3. For this design, we have $w=k=2, n_{c}=2$, $n_{f}=4$, and choose $\alpha=\beta=2$, resulting in $n_{0}=2, n_{\mathrm{W}}=4, \mathbf{u}_{\mathrm{W}}=\frac{1}{2} \mathbf{1}_{w}$, and $\mathbf{u}_{\mathrm{S}}=\frac{1}{2} \mathbf{1}_{k}$, and $\mathbf{V}_{\mathrm{S}}=\frac{n}{k} \mathbf{1}_{k} \mathbf{1}_{k}^{\prime}=2 \mathbf{1}_{k} \mathbf{1}_{k}^{\prime}$, where $n$ refers to the number of subplot runs in the whole-plot containing the subplot axial points.

A balanced version of this design is identical except that whole-plot number 10 contains 4 subplot runs. At first glance, the small reduction in design size may not appear to be significant. However, we suggest augmenting the base design by replication of whole-plot 
number 10 to enable a pure-error based estimate of whole-plot error variance and to increase the degrees of freedom for a pure-error estimate of subplot variance. For $d f_{w}$ degrees of freedom for the whole-plot error estimate, $d f_{w}+1$ replicates of whole-plot 10 are required. Therefore, we see that the reduction in design size is not merely 2 subplot runs, rather it is $2\left(d f_{w}+1\right)$. The smaller size of these replicated whole-plots should be more appealing to a practitioner who is contemplating the cost-benefit trade-off of performing replicated whole-plots. This is especially true in the context that we are considering where the subplot runs are costly.

When $k$ is not a power of 2 , an unbalanced design can be generated if we eliminate one set of whole-plots containing the whole-plot factor at its axial level. The resulting design retains the capability to estimate a complete second-order model, however it does not contain all the design points found in a classical central composite design. An attractive feature of this structure is that the distance to the axial points is unrestricted. For example, consider a design for $\mathrm{D}(1,3)$ in Table 4 . The form of $\mathbf{K}$ for this type of design requires a non-zero $\mathbf{M}_{\mathrm{W}}$, which is not found in a classical VKM construction. A general development of this form of $\mathbf{K}$ is not provided, however for the design in Table $4, \mathbf{K}$ is defined by $n_{0}=2, n_{\mathrm{W}}=8$, $\mathbf{u}_{\mathrm{W}}=-6, \mathbf{u}_{\mathrm{S}}=4 \mathbf{1}_{k}, \mathbf{V}_{\mathrm{S}}=2 \mathbf{1}_{k} \mathbf{1}_{k}^{\prime}, \mathbf{m}_{\mathbf{0}}=\mathbf{0}$, and $\mathbf{M}_{\mathrm{W}}=2 \mathbf{1}_{k}^{\prime}$. Note that this design has two whole-plots with eight subplot runs, one with six subplot runs, and one with two subplot runs.

A summary of unbalanced VKM CCD's is provided in Table 5. The allotment of subplot runs to the whole-plots is denoted by a whole-plot size followed by its frequency in parentheses. For example, the design in Table 4 is denoted by $8(2), 6(1), 2(1)$. The $\mathrm{D}(w, 3)$ entries are based on removing one set of whole-plot axial points, as previously discussed, and therefore require two fewer whole-plots than other designs for the same $w$. 
Table 4: VKM Method Unbalanced Central Composite Design for $\mathrm{D}(1,3)$. $\left(z_{1}\right.$ is a HTC factor, $x_{1}, x_{2}$, and $x_{3}$ are ETC factors, $\alpha$ is the distance to the axial points for the ETC factors.)

\begin{tabular}{crrrr} 
Whole-Plot & $z_{1}$ & $x_{1}$ & $x_{2}$ & $x_{3}$ \\
\hline 1 & -1 & 1 & -1 & -1 \\
& -1 & -1 & 1 & -1 \\
& -1 & -1 & -1 & 1 \\
& -1 & 1 & 1 & 1 \\
& -1 & -1 & -1 & -1 \\
& -1 & 1 & 1 & -1 \\
& -1 & 1 & -1 & 1 \\
& -1 & -1 & 1 & 1 \\
\hline 2 & 1 & 1 & -1 & -1 \\
& 1 & -1 & 1 & -1 \\
& 1 & -1 & -1 & 1 \\
& 1 & 1 & 1 & 1 \\
& 1 & -1 & -1 & -1 \\
& 1 & 1 & 1 & -1 \\
& 1 & 1 & -1 & 1 \\
& 1 & -1 & 1 & 1 \\
\hline 3 & 0 & $-\alpha$ & 0 & 0 \\
& 0 & $\alpha$ & 0 & 0 \\
& 0 & 0 & $-\alpha$ & 0 \\
& 0 & 0 & $\alpha$ & 0 \\
& 0 & 0 & 0 & $-\alpha$ \\
& 0 & 0 & 0 & $\alpha$ \\
\hline 4 & 0 & 0 & 0 & 0 \\
& 0 & 0 & 0 & 0 \\
\hline & & &
\end{tabular}


Table 5: Summary of Unbalanced Central Composite and Box-Behnken Equivalent Estimation Split-Plot Designs by Construction Method.

( $w$ is the number of HTC factors, $k$ is the number of ETC factors, $m$ is the number of whole-plots, $n$ is the number of subplot runs per whole-plot denoted by the whole-plot size with its frequency in parentheses, and $N$ is the total number of subplot runs.)

\begin{tabular}{|c|c|c|c|c|c|c|c|c|c|c|}
\hline \multicolumn{2}{|c|}{ Factors } & \multicolumn{3}{|c|}{ VKM-CCD } & \multicolumn{3}{|c|}{ VKM-BBD } & \multicolumn{3}{|c|}{ MWP-BBD } \\
\hline$w$ & $k$ & $m$ & $n$ (freq.) & $N$ & $m$ & $n$ (freq.) & $N$ & $m$ & $n$ (freq.) & $N$ \\
\hline 1 & 2 & 6 & $4(5), 2(1)$ & 22 & 4 & $4(3), 2(1)$ & 14 & 3 & $5(1), 4(2)$ & 13 \\
\hline 1 & 3 & 4 & $8(2), 6(1), 2(1)$ & 24 & 4 & $12(3), 2(1)$ & 38 & 3 & $13(1), 6(2)$ & 25 \\
\hline 1 & 4 & 6 & $8(5), 2(1)$ & 42 & 4 & $24(3), 2(1)$ & 74 & 3 & $25(1), 8(2)$ & 41 \\
\hline 2 & 2 & 10 & $4(9), 2(1)$ & 38 & 10 & $4(9), 2(1)$ & 38 & & & \\
\hline 2 & 3 & 8 & $6(1), 4(6), 2(1)$ & 32 & 10 & $12(9), 2(1)$ & 110 & & & \\
\hline 2 & 4 & 10 & $8(9), 2(1)$ & 74 & 10 & $8(9), 2(1)$ & 74 & & & \\
\hline 3 & 2 & 16 & $4(15), 2(1)$ & 62 & 20 & $4(19), 2(1)$ & 78 & & & \\
\hline 3 & 3 & 14 & $6(1), 4(12), 2(1)$ & 56 & 19 & $4(18), 2(1)$ & 74 & & & \\
\hline 3 & 4 & 16 & $8(15), 2(1)$ & 122 & 21 & $4(20), 2(1)$ & 82 & & & \\
\hline
\end{tabular}




\section{Unbalanced VKM Method BBD}

The Box-Behnken design is an attractive alternative to the central composite in the completely randomized context due to its competitive size and utilization of three-levels. In the split-plot context, the three-levels are particularly appealing for the HTC factors. In this section, we consider applying the VKM construction method to the BBD.

As a result of our construction philosophy to utilize the minimum number of whole-plots, the number of subplot runs per whole-plot increases with the number of subplot factors. These large whole-plots may not be practical in certain applications, thereby requiring a design with additional whole-plots of a smaller size. Nevertheless, in this section we assume that these minimum whole-plot designs are permissible and consider methods to reduce the size of the whole-plots containing replicated subplot runs.

To minimize the number of whole-plots, we construct a single whole-plot with the HTC factor set at its center level. The size of this whole-plot, for $f \leq 5$, is

$$
n_{\text {max }}=4\left(\begin{array}{c}
k \\
2
\end{array}\right)=2 k(k-1) .
$$

To achieve balance, the subplot designs are replicated as required in the other whole-plots. The general form of $\mathbf{K}$ and the conditions expressed in Equation (6) hold for the BBD case. Following a similar approach as the CCD, we summarize the forms of $\mathbf{W}_{\mathrm{Q}_{i j}}$ and $\mathbf{S}_{\mathrm{Q}_{i j}}$ found in each type of whole-plot in Table 6. For cases (1)-(3), the position of the ones will change depending on the factors involved, however the form is consistent. Note that case (3) is not present when $w=1$.

From case (c) and Equation (7), we see that $n_{0}=n_{c}$. From case (1), we find

$$
\begin{aligned}
n_{1}-n_{c} & =2 \rho \\
\rho & =\frac{n_{1}-n_{c}}{2}=\frac{2 k(k-1)-n_{c}}{2} .
\end{aligned}
$$


Table 6: VKM Method BBD Whole-Plot Types.

$\left(\mathbf{W}_{\mathrm{Q}_{i j}}\right.$ is the $j^{t h}$ row of the model matrix containing the WP pure quadratics, $\mathbf{S}_{\mathrm{Q}_{i j}}$ is the $j^{\text {th }}$ row of the model matrix containing the SP pure quadratics, $n_{i}$ is the number of subplot runs in the $i^{\text {th }}$ whole-plot)

\begin{tabular}{|c|c|c|c|}
\hline Case & $\mathbf{W}_{\mathrm{Q}_{i j}}$ & $\mathbf{S}_{Q_{i j}}$ & $n_{i}$ \\
\hline $\mathrm{c}$ & $\left.\begin{array}{lll}0 & \cdots & 0\end{array}\right]_{w}$ & $\left.\begin{array}{lll}0 & \cdots & 0\end{array}\right]_{k}$ & $n_{c}$ \\
\hline 1 & {$\left[\begin{array}{lll}0 & \cdots & 0\end{array}\right]_{w}$} & {$\left[\begin{array}{lllll}1 & 1 & 0 & \cdots & 0\end{array}\right]_{k}$} & $n_{1}=4\left(\begin{array}{c}k \\
2\end{array}\right)=2 k(k-1)$ \\
\hline 2 & $\left.\begin{array}{llll}1 & 0 & \cdots & 0\end{array}\right]_{u}$ & $\left.\begin{array}{llll}1 & 0 & \cdots & 0\end{array}\right]_{k}$ & $n_{2}$ \\
\hline 3 & {$\left[\begin{array}{lllll}1 & 1 & 0 & \cdots & 0\end{array}\right]$} & $\left.\begin{array}{lll}0 & \cdots & 0\end{array}\right]_{k}$ & $n_{3}$ \\
\hline
\end{tabular}

For case (2), we find

$$
\begin{gathered}
n_{2}-n_{0}=\lambda+\rho \\
\lambda=n_{2}-n_{0}-\rho .
\end{gathered}
$$

Similarly for case (3), we find

$$
\lambda=\frac{2 k(k-1)-n_{c}}{2}
$$

Note that if $n_{1}=n_{2}=n_{3}=2 k(k-1)$, then $\lambda=\rho$.

Consider a design for the $\mathrm{D}(1,4)$ case given in Table 7 . To construct this design, we begin with a balanced BBD consisting of 4 whole-plots of size 24 . In whole-plots 1 and 2, there are 3 replicates of the base subplot design to balance the design. The vector notation denotes two subplot runs for each row in whole-plots 1 and 2. As with the CCD, we reduce the size of the whole-plot containing all factors at their center level. The number of subplot runs is reduced by $22\left(d f_{w}+1\right)$, which is very significant due to the large whole-plot size. Moreover, it is apparent that the likelihood of running whole-plot replicates is improved considerably. We note that the extreme unbalancing in this design may not be desirable in many experimental circumstances, however it does illustrate the achievable reduction in subplot replication. 
Table 7: VKM Method Unbalanced Box-Behnken Design for $\mathrm{D}(1,4)$. ( $z_{1}$ is a HTC factor, $x_{1}, x_{2}, x_{3}$ and $x_{4}$ are ETC factors.)

\begin{tabular}{|c|c|c|c|c|c|c|c|c|c|c|c|}
\hline Whole-Plot & $z_{1}$ & $x_{1}$ & $x_{2}$ & $x_{3}$ & $x_{4}$ & Whole-Plot & $z_{1}$ & $x_{1}$ & $x_{2}$ & $x_{3}$ & $x_{4}$ \\
\hline \multirow[t]{12}{*}{1} & -1 & \pm 1 & 0 & 0 & 0 & \multirow[t]{24}{*}{3} & 0 & 0 & -1 & -1 & 0 \\
\hline & -1 & 0 & \pm 1 & 0 & 0 & & 0 & 0 & 1 & -1 & 0 \\
\hline & -1 & 0 & 0 & \pm 1 & 0 & & 0 & 0 & -1 & 1 & 0 \\
\hline & -1 & 0 & 0 & 0 & \pm 1 & & 0 & 0 & 1 & 1 & 0 \\
\hline & -1 & \pm 1 & 0 & 0 & 0 & & 0 & -1 & 0 & 0 & -1 \\
\hline & -1 & 0 & \pm 1 & 0 & 0 & & 0 & 1 & 0 & 0 & -1 \\
\hline & -1 & 0 & 0 & \pm 1 & 0 & & 0 & -1 & 0 & 0 & 1 \\
\hline & -1 & 0 & 0 & 0 & \pm 1 & & 0 & 1 & 0 & 0 & 1 \\
\hline & -1 & \pm 1 & 0 & 0 & 0 & & 0 & 0 & 0 & -1 & -1 \\
\hline & -1 & 0 & \pm 1 & 0 & 0 & & 0 & 0 & 0 & 1 & -1 \\
\hline & -1 & 0 & 0 & \pm 1 & 0 & & 0 & 0 & 0 & -1 & 1 \\
\hline & -1 & 0 & 0 & 0 & \pm 1 & & 0 & 0 & 0 & 1 & 1 \\
\hline \multirow[t]{14}{*}{2} & +1 & \pm 1 & 0 & 0 & 0 & & 0 & -1 & -1 & 0 & 0 \\
\hline & +1 & 0 & \pm 1 & 0 & 0 & & 0 & 1 & -1 & 0 & 0 \\
\hline & +1 & 0 & 0 & \pm 1 & 0 & & 0 & -1 & 1 & 0 & 0 \\
\hline & +1 & 0 & 0 & 0 & \pm 1 & & 0 & 1 & 1 & 0 & 0 \\
\hline & +1 & \pm 1 & 0 & 0 & 0 & & 0 & 0 & -1 & 0 & -1 \\
\hline & +1 & 0 & \pm 1 & 0 & 0 & & 0 & 0 & 1 & 0 & -1 \\
\hline & +1 & 0 & 0 & \pm 1 & 0 & & 0 & 0 & -1 & 0 & 1 \\
\hline & +1 & 0 & 0 & 0 & \pm 1 & & 0 & 0 & 1 & 0 & 1 \\
\hline & +1 & \pm 1 & 0 & 0 & 0 & & 0 & -1 & 0 & -1 & 0 \\
\hline & +1 & 0 & \pm 1 & 0 & 0 & & 0 & 1 & 0 & -1 & 0 \\
\hline & +1 & 0 & 0 & \pm 1 & 0 & & 0 & -1 & 0 & 1 & 0 \\
\hline & +1 & 0 & 0 & 0 & \pm 1 & & 0 & 1 & 0 & 1 & 0 \\
\hline & & & & & & \multirow[t]{2}{*}{4} & 0 & 0 & 0 & 0 & 0 \\
\hline & & & & & & & 0 & 0 & 0 & 0 & 0 \\
\hline
\end{tabular}


For the design in Table 7 , we have $w=1, k=4, n_{c}=2$, resulting in $n_{0}=2, n_{\mathrm{W}}=24$, $\mathbf{u}_{\mathrm{W}}=11, \mathbf{u}_{\mathrm{S}}=(11) \mathbf{1}_{k}, \mathbf{V}_{\mathrm{S}}=6 \mathbf{1}_{k} \mathbf{1}_{k}^{\prime}, \mathbf{m}_{\mathbf{0}}=\mathbf{0}$, and $\mathbf{M}_{\mathrm{W}}=\mathbf{0}$.

A potentially unattractive feature of the design in Table 7 is the replicated subplot designs in whole-plots 1 and 2. We can reduce the size of these whole-plots by adding a non-zero $\mathbf{M}_{\mathrm{W}}$ matrix to $\mathbf{K}$. A general development of this form of $\mathbf{K}$ is not provided, however to illustrate this approach consider the design in Table 8. The first two whole-plots now contain 8 subplot runs each with no replication. For this design, $\mathbf{K}$ is defined by $n_{0}=2, n_{\mathrm{W}}=8, \mathbf{u}_{\mathrm{W}}=-5$, $\mathbf{u}_{\mathrm{S}}=(11) \mathbf{1}_{k}, \mathbf{V}_{\mathrm{S}}=6 \mathbf{1}_{k} \mathbf{1}_{k}^{\prime}, \mathbf{M}_{\mathrm{W}}=-4 \mathbf{1}_{k}^{\prime}$, and $\mathbf{m}_{\mathbf{0}}=\mathbf{0}$

The unbalanced design in Table 8 has achieved a significant reduction with $m=4$ and $N=42$ compared to Table 7 with $m=4$ and $N=70$, and the balanced version, which requires $m=4$ and $N=96$. Even though the number of whole-plots has not been reduced, the reduction in design size and particularly the size of the whole-plots that are proposed to be replicated, makes this design an attractive alternative.

A summary table of unbalanced VKM BBD's is provided in Table 5. For $\mathrm{D}(1, k)$, these designs require the same or fewer whole-plots than their CCD competitors, however the larger whole-plot size may not be permissible in some applications. In contrast, the $\mathrm{D}(3, k)$ designs require additional whole-plots over the unbalanced CCD's.

\section{Unbalanced MWP Method BBD}

In this section, we propose a class of unbalanced equivalent estimation designs that requires a minimum number of whole-plots to configure a completely randomized BBD into a split-plot structure for the $w=1$ case. We begin with a balanced design constructed using the MWP method. This method does not require the subplot centers to be contained in a whole-plot by themselves, rather they can be included in other whole-plots and added one-by-one without 
Table 8: Minimum Size VKM Method Unbalanced Box-Behnken Design for D $(1,4)$. ( $z_{1}$ is a HTC factor, $x_{1}, x_{2}, x_{3}$ and $x_{4}$ are ETC factors.)

\begin{tabular}{crrrrr} 
Whole-Plot & $z_{1}$ & $x_{1}$ & $x_{2}$ & $x_{3}$ & $x_{4}$ \\
\hline 1 & $\mathbf{- 1}$ & $\pm \mathbf{1}$ & 0 & 0 & 0 \\
& $\mathbf{- 1}$ & 0 & $\pm \mathbf{1}$ & 0 & 0 \\
& $\mathbf{- 1}$ & 0 & 0 & $\pm \mathbf{1}$ & 0 \\
& $\mathbf{- 1}$ & 0 & 0 & 0 & $\pm \mathbf{1}$ \\
\hline 2 & $+\mathbf{1}$ & $\pm \mathbf{1}$ & 0 & 0 & 0 \\
& $+\mathbf{1}$ & 0 & $\pm \mathbf{1}$ & 0 & 0 \\
& $+\mathbf{1}$ & 0 & 0 & $\pm \mathbf{1}$ & 0 \\
& $+\mathbf{1}$ & 0 & 0 & 0 & $\pm \mathbf{1}$ \\
\hline 3 & 0 & 0 & -1 & -1 & 0 \\
& 0 & 0 & 1 & -1 & 0 \\
0 & 0 & -1 & 1 & 0 \\
& 0 & 0 & 1 & 1 & 0 \\
0 & -1 & 0 & 0 & -1 \\
0 & 1 & 0 & 0 & -1 \\
& 0 & -1 & 0 & 0 & 1 \\
0 & 1 & 0 & 0 & 1 \\
& 0 & 0 & 0 & -1 & -1 \\
0 & 0 & 0 & 1 & -1 \\
0 & 0 & 0 & -1 & 1 \\
& 0 & 0 & 0 & 1 & 1 \\
0 & -1 & -1 & 0 & 0 \\
0 & 1 & -1 & 0 & 0 \\
0 & -1 & 1 & 0 & 0 \\
0 & 1 & 1 & 0 & 0 \\
0 & 0 & -1 & 0 & -1 \\
& 0 & 0 & 1 & 0 & -1 \\
0 & 0 & -1 & 0 & 1 \\
0 & 0 & 1 & 0 & 1 \\
0 & -1 & 0 & -1 & 0 \\
0 & 1 & 0 & -1 & 0 \\
0 & -1 & 0 & 1 & 0 \\
0 & 1 & 0 & 1 & 0 \\
\hline 0 & 0 & 0 & 0 & 0 \\
0 & 0 & 0 & 0 & 0 \\
\hline & & & & &
\end{tabular}


disturbing the equivalence property of the design.

To define $\mathbf{K}$ for an MWP Method BBD, we set $\mathbf{V}_{\mathrm{S}}=\mathbf{0}$ in Equation (3). Based on this form of $\mathbf{K}$ and Equation (4), the conditions that must be satisfied, beyond those related to the HTC factors that are satisfied by the split-plot structure, are

$$
\begin{aligned}
\mathbf{1}_{n_{i}} \mathbf{1}_{n_{i}}^{\prime} \mathbf{S}_{\mathrm{Q}_{i}} & =\mathbf{1}_{n_{i}} \mathbf{m}_{\mathbf{0}}^{\prime}+\mathbf{W}_{\mathrm{Q}_{i}} \mathbf{M}_{\mathrm{W}} \\
n_{i} \mathbf{1}_{n} & =n_{0} \mathbf{1}_{n_{i}}+\mathbf{W}_{\mathrm{Q}_{i}} \mathbf{u}_{\mathrm{W}} \quad \forall i .
\end{aligned}
$$

The first condition is unchanged from the balanced case where $\mathbf{m}_{\mathbf{0}}$ and $\mathbf{M}_{\mathrm{W}}$ are defined in Table 1. The second is similar to the VKM unbalanced case, except that there are no conditions on the subplot pure quadratic terms. Recall the form of $\mathbf{u}_{\mathrm{W}}$ is $\lambda \mathbf{1}_{w}$, and with $w=1$ we have $\mathbf{u}_{\mathrm{W}}$ as a scalar equal to $\lambda$. We rewrite the second condition in Equation (10) for $w=1$ as

$$
n_{i}-n_{0}=\lambda \mathbf{W}_{\mathrm{Q}_{i j}} \quad \forall i, j
$$

where $\mathbf{W}_{\mathrm{Q}_{i}}$ is a $n \times 1$ vector for $w=1$ of the form $z_{1}^{2} \mathbf{1}_{n_{i}}$. Therefore, for a BBD we only need to consider the two possible values of $z_{1}^{2}$ as 0 and 1 . Table 9 summarizes the two cases of whole-plot types. For $z_{1}^{2}=0$, we see that $n_{0}=n_{1}=2 k(k-1)+n_{c}$. For $z_{1}^{2}=1$, we find

$$
\begin{aligned}
\lambda & =n_{2}-n_{0} \\
\lambda & =2 k-\left[2 k(k-1)+n_{c}\right] \\
\lambda & =-\left(2 k^{2}-4 k+n_{c}\right) .
\end{aligned}
$$

Consider a design for $\mathrm{D}(1,3)$ in Table 10 . This design was derived from a balanced design with $m=3, n=13$, and $N=39$. The unbalanced version maintains 3 whole-plots, however the overall size has been reduced to $N=26$, by removing the replicated design points in the first two whole-plots. This is a similar construction as the design in Table 8 except we have 
Table 9: MWP Method BBD Whole-Plot Types.

\begin{tabular}{|c|c|c|}
\hline Case & $\mathbf{W}_{\mathrm{Q}_{i j}}$ & $n_{i}$ \\
\hline 1 & 0 & $n_{1}=2 k(k-1)+n_{c}$ \\
\hline 2 & 1 & $n_{2}=2 k$ \\
\hline
\end{tabular}

now been able to include the subplot centers in whole-plot number 3 , instead of requiring an additional whole-plot. For the design in Table $10, \mathbf{K}$ is defined by $n_{0}=14, n_{\mathrm{W}}=6$, $\mathbf{u}_{\mathrm{W}}=-8, \mathbf{m}_{\mathbf{0}}^{\prime}=8 \mathbf{1}_{k}, \mathbf{M}_{\mathrm{W}}=-6 \mathbf{1}_{k}^{\prime}$, and $\mathbf{V}_{\mathrm{S}}=\mathbf{0}$.

Note that a completely randomized version of a BBD with $f=4$ contains $N=24+n_{c}$ design points. Therefore, the equivalent estimation design in Table 10 achieves the minimum number of whole-plots to support a second-order model and requires no additional subplot runs over the completely randomized version. In fact, this is a general result for MWP method BBD's for $\mathrm{D}(1, k), k \in\{2,3,4\}$.

A summary table of unbalanced MWP method BBD's is provided in Table 5. To achieve the minimum number of whole-plots, these designs require a large number of subplot runs per whole-plot, similar to the VKM method BBD's.

\section{Minimal Point Designs}

As previously discussed, in some experimental situations the cost of the whole-plots approaches that of the subplots and both are expensive. In this situation, it is desirable to simultaneously minimize the number of whole-plots and the total number of subplot runs. Therefore, a saturated or near-saturated designs might be considered. As in the CRD context, we recommend the use of the $\mathrm{CCD}$ or $\mathrm{BBD}$, unless they are cost prohibitive. In addition, we recommend a cost comparison to conducting a completely randomized experiment, as proposed by Bisgaard (2000), which provides increased precision of the whole-plot 
Table 10: MWP Method Unbalanced Box-Behnken Design for $\mathrm{D}(1,3)$.

( $z_{1}$ is a HTC factor, $x_{1}, x_{2}$, and $x_{3}$ are ETC factors.)

\begin{tabular}{crrrr} 
Whole-Plot & $z_{1}$ & $x_{1}$ & $x_{2}$ & $x_{3}$ \\
\hline 1 & -1 & -1 & 0 & 0 \\
& -1 & 1 & 0 & 0 \\
& -1 & 0 & -1 & 0 \\
& -1 & 0 & 1 & 0 \\
& -1 & 0 & 0 & -1 \\
& -1 & 0 & 0 & 1 \\
\hline 2 & 1 & -1 & 0 & 0 \\
& 1 & 1 & 0 & 0 \\
& 1 & 0 & -1 & 0 \\
& 1 & 0 & 1 & 0 \\
& 1 & 0 & 0 & -1 \\
1 & 0 & 0 & 1 \\
\hline 3 & 0 & 0 & -1 & -1 \\
& 0 & 0 & -1 & 1 \\
& 0 & 0 & 1 & -1 \\
0 & 0 & 1 & 1 \\
& 0 & -1 & -1 & 0 \\
& 0 & -1 & 1 & 0 \\
0 & 1 & -1 & 0 \\
& 0 & 1 & 1 & 0 \\
0 & -1 & 0 & -1 \\
0 & -1 & 0 & 1 \\
0 & 1 & 0 & -1 \\
& 0 & 1 & 0 & 1 \\
0 & 0 & 0 & 0 \\
0 & 0 & 0 & 0 \\
\hline & & &
\end{tabular}


factors, versus the split-plot structure.

Goos (2002) highlights that all saturated designs are equivalent. For a saturated design, we have $N$ design points to estimate a model with $p$ parameters where $N=p$. Therefore, the model matrix, $\mathbf{X}$ is a square full rank matrix. The OLS estimate is

$$
\hat{\boldsymbol{\beta}}_{O L S}=\left(\mathbf{X}^{\prime} \mathbf{X}\right)^{-1} \mathbf{X}^{\prime} \mathbf{y}=\mathbf{X}^{\prime-1}\left(\mathbf{X}^{\prime}\right)^{-1} \mathbf{X}^{\prime} \mathbf{y}=\mathbf{X}^{-1} \mathbf{y}
$$

The GLS estimate is

$$
\hat{\boldsymbol{\beta}}_{G L S}=\left(\mathbf{X}^{\prime} \boldsymbol{\Sigma}^{-1} \mathbf{X}\right)^{-1} \mathbf{X}^{\prime} \boldsymbol{\Sigma}^{-1} \mathbf{y}=\mathbf{X}^{-1} \boldsymbol{\Sigma}\left(\mathbf{X}^{\prime}\right)^{-1} \mathbf{X}^{\prime} \boldsymbol{\Sigma}^{-1} \mathbf{y}=\mathbf{X}^{-1} \mathbf{y}
$$

which is the same as the OLS estimate. Even though all saturated designs are equivalent, some are more suitable for augmentation to provide pure-error estimates of the variance components and lack-of-fit degrees of freedom while maintaining the equivalence property.

In the previous sections, design construction was based on an analytical derivation of the form of the $\mathbf{K}$ matrix to satisfy the equivalence condition for a particular design structure. As previously mentioned, Equation (2) can also be used to numerically test the equivalence of any arbitrary split-plot design without knowledge of the form of $\mathbf{K}$. If the design is found to be equivalent, then $\mathbf{K}$ can be computed directly from Equation (1). Computing K provides insight into permissible augmentation strategies that maintain the equivalence property. For example, we can determine if the design can be augmented by adding subplot centers one-by-one or a whole-plot containing all factors at their center level.

Many of these minimal point designs feature non-orthogonal subplot designs. While orthogonality is desirable and was maintained in the CCD and BBD constructions, these designs demonstrate the flexibility in generating equivalent estimation designs. In addition, to obtain minimal point split-plot designs, we have relaxed our constraint of $n_{\min }=2$, allowing whole-plots of size one, which are typically undesirable for HTC factors. 
Table 11: Notz Saturated Design Design for $\mathrm{D}(1,3)$.

$\left(z_{1}\right.$ is a HTC factor, $x_{1}, x_{2}$, and $x_{3}$ are ETC factors.)

\begin{tabular}{crrrr} 
Whole-Plot & $z_{1}$ & $x_{1}$ & $x_{2}$ & $x_{3}$ \\
\hline 1 & -1 & 1 & 1 & -1 \\
& -1 & 1 & -1 & 1 \\
& -1 & -1 & 1 & 1 \\
& -1 & 1 & 1 & 1 \\
& -1 & -1 & -1 & -1 \\
\hline 2 & 1 & 1 & -1 & -1 \\
& 1 & -1 & 1 & -1 \\
& 1 & -1 & -1 & 1 \\
& 1 & 1 & 1 & -1 \\
& 1 & 1 & -1 & 1 \\
& 1 & -1 & 1 & 1 \\
& 1 & 0 & 0 & 0 \\
\hline 3 & 0 & 1 & 0 & 0 \\
& 0 & 0 & 1 & 0 \\
& 0 & 0 & 0 & 1 \\
\hline
\end{tabular}

In this section, we consider the minimal point designs proposed by Notz (1982) to illustrate the utility of the numerical verification procedure. Consider a saturated Notz design for $\mathrm{D}(1,3)$ in Table 11 . This design can be augmented to provide 1 degree of freedom for lack-of-fit $\left(d f_{L o F}\right)$, by including a subplot center run to whole-plot number 3 , or by adding a subplot run to whole-plot number 2 with all of the subplot factors at their low level, $\left[\begin{array}{lll}-1 & -1 & -1\end{array}\right]$. Both of these augmentations can be verified to possess the equivalence property using Equation (2). A similar procedure can be employed for other numbers of factors. When the augmentation is not intuitive, a $\mathcal{D}$-optimal augmentation strategy was applied to add the minimum number of design points while maintaining the equivalence property. Table 12 contains a summary of Notz equivalent estimation split-plot designs.

Following a similar approach, equivalent estimation split-plot versions of the Hoke (1974), Box and Draper (1974), hybrid proposed by Roquemore (1976), and small composite pro- 
Table 12: Summary of Notz Equivalent Estimation Split-Plot Designs.

( $w$ is the number of HTC factors, $k$ is the number of ETC factors, $m$ is the number of whole-plots, $n$ is the number of subplot runs per whole-plot denoted by the whole-plot size with its frequency in parentheses, $N$ is the total number of subplot runs, and $d f_{\text {LoF }}$ is the number of lack-of-fit degrees of freedom.)

\begin{tabular}{|c|c|c|c|c|c|c|c|c|}
\hline \multicolumn{2}{|c|}{ Factors } & \multicolumn{3}{|c|}{ Saturated } & \multicolumn{4}{|c|}{ Augmented } \\
\hline$w$ & $k$ & $m$ & $n$ (freq.) & $N$ & $m$ & $n$ (freq.) & $N$ & $d f_{L o F}$ \\
\hline 1 & 1 & 3 & $3(1), 2(1), 1(1)$ & 6 & 3 & $3(1), 2(2)$ & 7 & 1 \\
\hline 1 & 2 & 3 & $5(1), 3(1), 2(1)$ & 10 & 3 & $5(1), 4(1), 2(1)$ & 11 & 1 \\
\hline 1 & 3 & 3 & $7(1), 5(1), 3(1)$ & 15 & 3 & $7(1), 5(1), 4(1)$ & 16 & 1 \\
\hline 1 & 4 & 3 & $11(1), 8(1), 2(1)$ & 21 & & & & \\
\hline 2 & 1 & 7 & $2(3), 1(4)$ & 10 & 7 & $2(4), 1(3)$ & 11 & 1 \\
\hline 2 & 2 & 7 & $3(3), 2(2), 1(2)$ & 15 & 7 & $4(1), 3(2), 2(2), 1(2)$ & 16 & 1 \\
\hline 2 & 3 & 7 & $6(1), 4(3), 1(3)$ & 21 & & & & \\
\hline 2 & 4 & 7 & $9(1), 5(3), 2(1), 1(2)$ & 28 & 7 & $9(1), 5(3), 3(1), 2(1), 1(1)$ & 30 & 2 \\
\hline 3 & 1 & 12 & $2(3), 1(9)$ & 15 & 12 & $2(8), 1(4)$ & 20 & 5 \\
\hline 3 & 2 & 12 & $2(9), 1(3)$ & 21 & & & & \\
\hline 3 & 3 & 12 & $4(1), 3(4), 2(5), 1(2)$ & 28 & & & & \\
\hline
\end{tabular}


posed by Hartley (1959), Draper (1985) and Draper and Lin (1990) minimal point response surface designs have been constructed.

The ability to numerically verify that a design provides equivalent estimates using Equation (2) has proven useful in exploring other families of RSM designs that do not provide a consistent analytical form of $\mathbf{K}$, for example classes of $\mathcal{D}$-optimal designs. In addition, numerical verification provides a convenient method to determine if model projection has disturbed the equivalence property.

\section{Concluding Remarks}

In this paper, we have relaxed the assumption of balance and demonstrated that unbalanced equivalent estimation split-plot designs can be constructed from families of completely randomized response surface designs. We have shown how to start with a balanced design and reduce the size of the whole-plots that contain subplot replicates. These unbalanced designs are applicable when the subplot cost becomes relatively expensive, approaching the cost of the whole-plots, and the experimental apparatus is suitable for unequal whole-plot sizes.

The construction methods discussed in this paper provide a flexible approach to transform completely randomized response surface designs into a split-plot structure that provides best linear unbiased estimates and simplified estimation. A comprehensive catalog containing split-plot versions of many classical response surface designs is available at the author's website, and includes balanced and unbalanced versions. The straightforward and intuitive nature of these construction methods coupled with the pre-built catalog makes equivalent estimation split-plot designs an attractive option for practitioners. 


\section{References}

[1] Bisgand, S. (2000). "The Design and Analysis of $2^{k-p} \times 2^{q-r}$ Split Plot Experiments." Journal of Quality Technology 32, pp. 39-56.

[2] Box, G. E. P. and Behnken, D. W. (1960). "Some New Three-Level Designs for the Study of Quantitative Variables". Technometrics 2, pp. 455-475.

[3] Box, G. E. P. and Draper, N. R. (1974). "Some Minimum Point Designs for Second Order Response Surfaces". Technometrics 16, pp. 613-616.

[4] Box, G. E. P. and Draper, N. R. (1975). "Robust Designs". Biometrika 62, (2), pp. $347-352$.

[5] Box, G. E. P. and Wilson, K. B. (1951). "On the Experimental Attainment of Optimum Conditions". Journal of the Royal Statistical Society Ser.B, 13, pp. 195-241.

[6] Draper, N. R. (1985), "Small Composite Designs," Technometrics, 27, 173-180.

[7] Draper, N. R. and John, J. A. (1998). "Response Surface Designs where Levels of Some Factors Are Difficult to Change". Australian and New Zealand Journal of Statistics 40, pp. 487-495.

[8] Draper, N. R. and Lin, D. K. J. (1990), "Small Response Surface Designs," Technometrics, 32, 187-194.

[9] Goos, P. (2002). The Optimal Design of Blocked and Split-Plot Experiments. SpringerVerlag, New York.

[10] Goos, P. and Vandebroek, M. (2001). "Optimal Split-Plot Designs". Journal of Quality Technology 33, pp. 436-450.

[11] Goos, P. and Vandebroek, M. (2003). "D-optimal Split-Plot Designs with Given Numbers and Sizes of Whole Plots". Technometrics 45, pp. 235-245.

[12] Graybill, F. A. (1976). Theory and Application of the Linear Model. Duxbury, North Scituate, MA.

[13] Hartley, H. O. (1959). "Small Composite Design for Quadratic Response Surfaces." Biometrika 15, pp. 611-624. 
[14] Hoke, A. T. (1974). "Economical Second Order Designs Based on Irregular Fractions." Technometrics 17, pp. 375-384.

[15] Letsinger, J. D. ; Myers, R. H. ; and Lentner, M. (1996). "Response Surface Methods for Bi-Randomization Structures". Journal of Quality Technology 28, pp. 381397.

[16] McElroy, F. W. (1967). "A Necessary and Sufficient Condition that Ordinary Least Squares Estimators Be Best Linear Unbiased". Journal of the American Statistical Association, 62, pp. 1302-1304.

[17] Montgomery, D. C. (2004). Design and Analysis of Experiments 6th edition. John Wiley \& Sons, New York, NY.

[18] Myers, R. H. and Montgomery, D. C. (2002). Response Surface Methodology 2nd edition. John Wiley \& Sons, New York, NY.

[19] Notz, W. (1982). "Minimal Point Second Order Designs". Journal of Statistical Planning and Inference. 6, pp. 47-58.

[20] Parker, P. A. , Kowalski, S. M. and Vining, G. G. (2005a), "Construction of Balanced Equivalent Estimation Second-Order Split-Plot Designs," submitted to Technometrics.

[21] Parker, P. A., Kowalski, S. M. and Vining, G. G. (2005b), "Classes of Split-Plot Response Surface Designs for Equivalent Estimation," submitted to Quality and Reliability Engineering International.

[22] Roquemore, K. G. (1976). "Hybrid Designs for Quadratic Response Surfaces". Technometrics 18 , pp. 419-423.

[23] Trinca, L. A. and Gilmour, S. G. (2001). "Multistratum Response Surface Designs". Technometrics 43, pp. 25-33.

[24] Vining, G. G. ; Kowalski, S. M. ; and Montgomery, D. C. (2005) "Response Surface Designs Within a Split-Plot Structure". Journal of Quality Technology 37, pp. 115-129. 